\title{
Saúde bucal e autopercepção em adultos e idosos de Piracicaba, SP
}

\author{
SILVA, Débora Dias da* \\ CARVALHO, Osvaldo Luis de ex** \\ SOUSA, Maria da Luz Rosário de ${ }_{* * *}$ \\ HEBLING, Eduardo
}

\begin{abstract}
RESUMO
O objetivo deste trabalho foi verificar as condições de saúde bucal, bem como as condições sócioeconômicas, de acesso a serviços odontológicos e autopercepção da saúde bucal em idosos e adultos no município de Piracicaba (SP). A amostra foi de 23 idosos (65 a 74 anos) e 54 adultos ( 35 a 44 anos). Os exames foram realizados em domicílios, de acordo com critérios da Organização Mundial de Saúde. Encontrou-se 52,2\% de edentulismo para os idosos e 2,0\% para os adultos, sendo a média de dentes presentes de 6,9 e 20,7, respectivamente. Os idosos apresentaram CPOD de 27,0 e os adultos 19,9. O maior componente do índice CPOD foi dentes perdidos no grupo de idosos $(92,3 \%)$ e adultos $(52 \%)$. Quanto à necessidade de próteses totais, somente $5,6 \%$ dos adultos necessitavam de superiores e nenhum de inferiores, dos idosos, $17,4 \%$ tinham necessidade de próteses superiores e $21,7 \%$ de inferiores. Quanto aos demais resultados (características socioeconômicas, acesso a serviço odontológico e autopercepção), houve diferença entre os grupos apenas com relação a escolaridade, onde os adultos apresentaram melhores condições. A autopercepção foi semelhante entre os grupos, com dados positivos, apesar das condições clínicas serem diferentes. Diante destes resultados, há necessidade de programas preventivos e educativos tanto para idosos como para adultos, para que estes adultos cheguem à terceira idade com uma condição de saúde bucal melhor que a relatada atualmente.
\end{abstract}

PALAVRAS-CHAVE:

Adultos. Idosos. Saúde Bucal. Autopercepção. Levantamentos de saúde bucal.

\section{INTRODUÇ̃̃ O}

Os levantamentos epidemiológicos são importantes, pois oferecem base para que se possa estimar a situação atual e as futuras necessidades de cuidados de saúde bucal em uma população (WORLD HEALTH ORGANIZATION (WHO), 1987).

E por se tratar de população, torna-se necessário ressaltar um fato atual que é o envelhecimento desta, evidenciado pelo crescente aumento da população idosa em todo o mundo, comprovado através de numerosos estudos demográficos (PAPALLÉO NETTO; PONTES, 2002).

No entanto, paralelamente às modificações demográficas, há necessidade também de profundas transformações socioeconômicas (particularmente nos países em desenvolvimento), visando melhor qualidade de vida aos idosos e àqueles que se encontram em processo de envelhecimento, que são os adultos (PAPALLÉO NETTO; PONTES, 2002).

Assim, para avaliar a qualidade de vida, os levantamentos epidemiológicos têm sido complementados com questões sobre a autopercepção dos indivíduos, visando um melhor entendimento do que é sentido e quais as necessidades percebidas em relação à saúde.
Os adultos constituem a larga maioria da população, que demandam fortemente por serviços odontológicos e influenciam decisivamente o comportamento de seus dependentes (Pinto, 2000), porém, há escassez de relatos sobre saúde bucal referente a esta população.

Acrescenta-se ainda que, paralelamente à cárie e às doenças periodontais, ainda é comum a prática de extrações em série, o que conduz ao edentulismo e ao uso de próteses. $\mathrm{E}$ em se tratando de Brasil, é necessário ressaltar que existe uma dificuldade de acesso aos serviços odontológicos por uma grande parte dos indivíduos (PINTO, 2000; BRASIL, 1998).

Outro fator importante a se destacar, é o papel dos fatores sócio-comportamental e ambiental nas doenças bucais e saúde e isto tem sido demonstrado em vários levantamentos epidemiológicos (PETERSEN, 2003). No geral, as cidades com níveis mais altos de prevalência de cárie, também apresentam um perfil com renda insuficiente; adultos sem alfabetização; menor renda familiar além de menor IDH (Indice de Desenvolvimento Humano) (PERES et al., 2003).

Como não se tem conhecimento das reais condições da saúde bucal da população adul- ta e idosa, pois a atenção à saúde bucal não abrange todos os grupos populacionais (SHINKAI; DEL BEL CURY, 2000), o objetivo deste estudo foi verificar a prevalência de cárie, enfatizando o número de dentes presentes, uso e necessidade de próteses totais e edentulismo, descrevendo a autopercepção, acesso a serviços odontológicos e fatores sócio-econômicos em idosos e adultos no Município de Piracicaba, para que através do conhecimento destas condições, possam ser estabelecidas ações específicas direcionadas a estes grupos.

\section{MATERIAIS E MÉTODOS}

Este foi um estudo transversal, denominado Condições de Saúde Bucal no Estado de São Paulo em 2002 (SÃO PAULO, 2002), sendo parte de um projeto de abrangência nacional, denominado Projeto SB Brasil (BRASIL, 2004). A SES-SP(Secretaria do Estado de Saúde de São Paulo) e a FSP-SP (Faculdade de Saúde Pública da Universidade de São Paulo) coordenaram o projeto com ampliação da amostra para o Estado de São Paulo, além de contarem com a participação de 7 Faculdades de Odontologia do Estado (SÃO PAULO, 2002).

* Programa de Pós Graduação em Odontologia, Área de Cariologia Faculdade de Odontologia de Piracicaba da Universidade Estadual de Campinas. ** Mestrado Profissionalizante, Área de Saúde Coletiva da Faculdade de Odontologia de Piracicaba da Universidade Estadual de Campinas. *** Departamento de Odontologia Social da Faculdade de Odontologia de Piracicaba da Universidade Estadual de Campinas. 
Para a amostra correspondente ao Estado de São Paulo, foram sorteados 7 municípios por porte, totalizando 35 municípios segundo os 5 portes, com o seguinte número de habitantes: até $5.000 ;$ de 5.001 a $10.000 ;$ de 10.001 a 50.000; de 50.001 a 100.000 e mais de 100.000 habitantes. Dentre os municípios sorteados, Piracicaba correspondeu a um dos com mais de 100.000 habitantes.

Foi adotado como base para o tamanho amostral em cada grupo etário, a variável cárie dentária, medida pelo índice CPOD (número médio de dentes afetados pelos indivíduos). Para os adultos e idosos, o tamanho da amostra foi calculado para cada macrorregião no país (Sul, Sudeste, Centro-Oeste, Norte e Nordeste), a partir das estimativas de cárie produzidas em 1986, pelo Ministério da Saúde (BRASIL, 1988). Ressalta-se que não foram utilizados dados mais recentes (Levantamento Epidemiológico em Saúde Bucal: Estado de São Paulo, 1998) (SÃO PAULO, 1999) para o cálculo do tamanho da amostra, em função da adoção do mesmo critério metodológico utilizado para o estudo nacional (BRASIL, 2004).

Assim, a amostra foi probabilística e estratificada por idade, tendo representatividade tanto nacional, quanto estadual e municipal, de acordo com os parâmetros estabelecidos pelo levantamento, onde o total de indivíduos examinados foram 54 adultos $(35$ a 44 anos) e 23 idosos (65 a 74 anos), para o município de Piracicaba.

O processo de calibração (treinamento) foi planejado considerando-se 05 examinadores/ município. Todo o processo de calibração da equipe foi dimensionado para abranger, pelo menos, 24 horas de trabalho.

A concordância intra-examinadores foi verificada durante o processo de calibração $(99,0 \%$, com IC95\% entre 98,1 e 99,5) e durante a fase de coleta de dados a partir do exame em duplicata em $10 \%$ da amostra (com valores acima de 95\%). A concordância interexaminadores, aferida no processo de calibração foi de 98,9\%, com IC95\% entre 98,0 e 99,4, considerados como valores aceitáveis para estudos epidemiológicos.

Os índices utilizados obedeceram aos códigos e critérios recomendados pela Organização Mundial da Saúde (OMS) (WHO, 1997). As seguintes condições foram pesquisadas: cárie dentária (índice CPOD), uso e necessidade de próteses totais. Foram também obtidos dados relativos a condições socioeconômicas, acesso a serviços odontológicos e autopercepção da saúde bucal (Quadro 1). Ressalta-se que no presente estudo foram avaliadas somente as questões com asterisco.

A coleta de dados ocorreu durante os meses de maio a julho de 2002 , sendo que os exames intrabucais foram realizados em unidades domiciliares e seguiram a metodologia proposta pela OMS (WHO, 1997). Foram utilizados espelhos bucais planos e sondas periodontais preconizadas OMS, sob iluminação natural, ventilação e proximidade de uma fonte de água, onde o examinador e o indivíduo a ser examinado deveriam estar sentados.

Para a análise dos dados, foi utilizado o teste Qui quadrado e Exato de Fischer para verificar as diferenças das variáveis entre os grupos, usando os valores numéricos.

Por se tratar de uma pesquisa que envolveu seres humanos, a mesma foi submetida e aprovada pelo Comitê de Ética em Pesquisa

\section{Quadro 1: Avaliação sócioeconômica, acesso e autopercepção em saúde bucal.}

\section{CARACTERIZAÇÃO SÓCIOECONÔMICA}

1- número de pessoas na casa

*2- escolaridade (anos de estudo)

3- estudante

0 - Sim

4- tipo de escola

0 - não é estudante

3 - outros

5- moradia

1 - própria

4 - cedida

6 - número de cômodos na casa

7 - renda familiar (em reais)

*8 - renda pessoal (em reais)

*9 - posse de automóvel

0 - não possui

1 - Possui 1 automóvel

2 - Possui 2 ou + automóveis

ACESSO A SERVIÇOS ODONTOLÓGICOS

* 10 - Já foi ao dentista alguma vez na vida?

0 - Sim 1 - Não

* 11 - Há quanto tempo?

0 - Nunca foi ao dentista

$3-3$ ou + anos

12- Onde?

0 - Nunca foi ao dentista 1 - Serviço público

3 - Serviço privado (planos e convênios)

5 - Outros

13- Por quê?

0 - Nunca foi ao dentista 1 - Consulta de rotina/reparos/manutenção

2 - Dor

4 - Cavidades nos dentes 5 - feridas, caroços ou manchas na boca

6 - rosto inchado

14- Como avalia o atendimento?

0 - Nunca foi ao dentista 1 - Péssimo 2 - Ruim
3 - Regular
4- Bom
5 - Ótimo

* 15 - recebeu informaç̃es sobre como evitar problemas bucais?

0 - Sim 1 - Não

*16- Considera que necessita de tratamento atualmente?

0 - Sim 1 - Não

AUTOPERCEPCÃO DA SAÚDE BUCAL

* 17 - Como classificaria sua saúde bucal?

0 - Não sabe/não informou 1- Péssima 2- Ruim

3-Regular 4-Boa 5- Ótima

* 18 - Como classificaria a aparência de seus dentes e gengivas?

0 - Não sabe/não informou 1 - Péssima 2 - Ruim

3 - Regular 4- Boa 5- Ótima

* 19 - Como classificaria a sua mastigação?

0- Não sabe/ não informou 1 - Péssima 2 - Ruim

3 - Regular 4 - Boa 5 - Ótima

* 20 - Como classificaria a sua fala devido aos seus dentes e gengivas?

0 - Não sabe/ não informou 1 - Péssima 2 - Ruim
3 - Regular
4- Boa
5 - Ótima

*21 - De que forma a sua saúde bucal afeta o seu relacionamento com outras pessoas?

0 - Não sabe/não informou 1 - Não afeta 2 - Afeta pouco

3 - Afeta mais ou menos 4-Afeta muito

*22 - 0 quanto de dor seus dentes e gengivas causaram nos últimos 6 meses?

0 - Nenhuma dor 1 - Pouca dor 2 - Média dor

3 - Muita dor
Te Odontologia de Piracicaba com o protocolo 154/

Do total da amostra, a maioria dos idosos a $(65,2 \%)$ eram mulheres e quanto à etnia, a maioria dos idosos e dos 
adultos eram brancos, com percentual de $87 \%$ e $85,2 \%$, respectivamente.

Os idosos apresentaram CPOD médio de 27,0, com a maior porcentagem relativa a dentes perdidos $(92,3 \%)$. Já o CPOD relativo aos adultos foi de 19,9, onde a maior porcentagem também foi de dentes perdidos (52\%), seguida de dentes restaurados $(39,3 \%)$ As médias do CPOD e seus componentes podem ser visualizadas na Tabela 1 .

A média de dentes hígidos foi de 5,0 para os idosos e 12,1 para os adultos; quanto aos dentes presentes, a média foi de 6,9 e 20,7 , respectivamente. Torna-se importante ressaltar que $52,2 \%(n=12)$ dos idosos e 2,0\% (n=1) dos adultos eram edêntulos. [Tabela 2]. Os que apresentaram 20 ou mais dentes presentes foram $13,0 \%(n=3)$ dos idosos e $64,8 \%(n=35)$ dos adultos. vam próteses totais superiores e 39,1\% (n=9) inferiores, dos adultos, somente $16,7 \%(n=9)$ e 2,0\% (n=1) usavam próteses totais superiores e inferiores, respectivamente. [Tabela 2]

Com relação aos idosos, a necessidade de próteses totais foi de $17,4 \%(\mathrm{n}=4)$ para superior e de $21,7 \%(n=5)$ para a inferior, para os adultos, esta necessidade foi de apenas $5,6 \%$ $(n=3)$ para a arcada superior e sem necessidade para a inferior. [Tabela 2]

As condições socioeconômicas tanto dos adultos como dos idosos não foram muito diferentes, onde $55,6 \%$ dos adultos e $47,8 \%$ dos idosos não possuíam renda pessoal e a maioria (59,3\% dos adultos e $69,6 \%$ dos idosos) não tinha automóvel. [Tabela 3]

Quanto à escolaridade, 35\% (n=19) dos adultos tiveram de 1 a 4 anos de escolaridade e

Tabela 1 - Experiência de cárie (CPOD e componentes) em adultos e idosos. Piracicaba, 2002.

\begin{tabular}{|c|c|c|c|}
\hline \multirow{2}{*}{$\begin{array}{l}\text { Variáveis } \\
\text { (média) }\end{array}$} & \multicolumn{2}{|c|}{ Grupos } & \multirow{2}{*}{$\mathbf{P}$} \\
\hline & adultos & idosos & \\
\hline Dentes cariados & 1,7 & 1,3 & - \\
\hline Dentes perdidos & 10,4 & 24,9 & $<0,05$ \\
\hline Dentes restaurados & 7,8 & 0,8 & - \\
\hline CPOD (desvio padrão) & $19,9(6,7)$ & $27,0(7,5)$ & - \\
\hline
\end{tabular}

Nota: foi utilizado o teste Qui quadrado para verificar a diferença entre os grupos para a variável número de dentes perdidos.

Da amostra de idosos, $61,0 \%(n=14)$ usa-

dos idosos, $78,2 \%(\mathrm{n}=18)$ freqüentaram a escola num período que variou de 2 a 4 anos. Somente os adultos apresentaram escolaridade acima de 9 anos $(33,5 \%)$, revelando assim melhor escolaridade para os adultos $(\mathrm{p}=0,04)$. [Tabela 3]

A grande parte dos adultos $(96,3 \%)$ e todos os idosos já foram ao dentista alguma vez; quanto ao tempo da última visita ao dentista, $56,5 \%$ dos idosos foram há 3 anos ou mais e 46,3\% dos adultos há menos de 1 ano. A maioria (idosos e adultos) foi informada de como evitar problemas bucais e considera que tem necessidade de tratamento. [Tabela 4]

Quanto a autopercepção da saúde bucal, entre os adultos, a minoria $(31,5 \%)$ classificou a saúde bucal como ruim ou péssima, bem como a aparência dos dentes e gengivas; muitos $(46,3 \%)$ responderam que têm boa mastigação e quanto à fala, metade $(50 \%)$ relatou

Tabela 2 - Variáveis clínicas em adultos e idosos. Piracicaba, 2002.

\begin{tabular}{|c|c|c|c|}
\hline \multirow{2}{*}{ Variáveis } & \multicolumn{2}{|c|}{ Grupos } & \multirow{2}{*}{$\mathbf{P}$} \\
\hline & adultos & idosos & \\
\hline Dentes hígidos (média) & 12,1 & 5,0 & $<0,05$ \\
\hline Edentulismo (\%) & 2,0 & 52,2 & - \\
\hline Dentes presentes (média) & 20,7 & 6,9 & - \\
\hline Presença de 20 + dentes $(\%)$ & 64,8 & 13 & $<0,05$ \\
\hline \multicolumn{4}{|l|}{ Uso de prótese total $(\%)$} \\
\hline Superior & 16,7 & 61,0 & - \\
\hline Inferior & 2,0 & 39,1 & - \\
\hline \multicolumn{4}{|l|}{ Necessidade de prótese total } \\
\hline Superior & 5,6 & 17,4 & - \\
\hline Inferior & 0 & 21,7 & - \\
\hline
\end{tabular}

Nota: foi utilizado o teste Qui quadrado para verificar a diferença entre os grupos para as variáveis: número de dentes hígidos e presença de $20 \mathrm{ou}+$ dentes. que esta era classificada como boa. Já a maior parte foi a que respondeu que a saúde bucal não influencia em seus relacionamentos $(57,4 \%)$ e também os que não tiveram dor em seus dentes e gengivas nos últimos 6 meses $(53,7 \%)$. [Tabela 5]

Os idosos perceberam sua saúde bucal da mesma forma que os adultos, a minoria classificou sua saúde bucal $(17,3 \%)$, a aparência dos dentes e gengivas $(21,7 \%)$ e a mastigação $(26,1 \%)$ como ruim ou péssima; a fala foi classificada como boa por muitos $(39,1 \%)$ e a maioria relatou que a saúde bucal não afeta seus relacionamentos $(56,5 \%)$ e que não teve dor nos dentes e gengivas nos últimos 6 meses $(60,9 \%)$, sem diferenças significantes entre os grupos $(p>0,05)$. [Tabela 5]

\section{DISCUSSÃ O}

A grande importância deste estudo deveuse ao fato de se tratar de um estudo domiciliar, com dados complementares e inovadores em levantamentos epidemiológicos em saúde bucal, com abordagem de fatores socioeconômicos, acesso a serviços e autopercepção da saúde bucal.

Dados atuais referentes às Condições de Saúde Bucal no Estado de São Paulo (SÃO PAULO, 2002) em que o município de Piracicaba foi inserido na amostra $(\mathrm{CPOD}=19,9$ para adultos), revelaram experiência de cárie nos adultos de 20,3 dentes, onde, apesar da amostra ter sido domiciliar, não se apresentou muito diferente da encontrada no Estado de São Paulo no ano de 1998, em que os adultos apresentaram 22,39 dentes com experiência de cárie, sendo a perda dentária responsável por $50 \%$ do valor total deste índice (FRAZÃO et al., 2003).

Complementando os dados sobre nível sócio-econômico, em Piracicaba o IDH (Índice de Desenvolvimento Humano) para o ano de 2000 era de 0,836, muito próximo do Estado de São Paulo (0,814), sendo considerado como alto desenvolvimento humano (FUNDAÇÃO SISTEMA ESTADUAL DE ANÁLISE DE DADOS, 2005), o que pode estar refletindo condições semelhantes para estas populações.

Quanto aos idosos, no Estado de São Paulo a experiência de cárie foi de 28,6 em 1998 e 
Tabela 3 - Respostas relativas às questões sobre caracterização socioeconômica em adultos e idosos. Piracicaba, 2002.

\begin{tabular}{|c|c|c|c|}
\hline \multirow{2}{*}{ Variáveis } & \multicolumn{2}{|c|}{ Grupos } & \multirow{2}{*}{$\mathbf{P}$} \\
\hline & adultos & idosos & \\
\hline \multicolumn{4}{|l|}{ Escolaridade (\%) } \\
\hline Nenhuma & 2,0 & 13,0 & \\
\hline De 1 a 4 anos & 35,0 & 78,2 & $<0,05^{1}$ \\
\hline De 5 a 9 anos & 29,5 & 8,8 & \\
\hline Acima de 9 anos & 33,5 & - & \\
\hline \multicolumn{4}{|l|}{ Renda pessoal $(\%)$} \\
\hline Não possui & 55,6 & 47,8 & \\
\hline Até $R \$ 300,00$ & 20,2 & 34,7 & $>0,05^{2}$ \\
\hline Acima de $R \$ 300,00$ & 24,2 & 17,5 & \\
\hline \multicolumn{4}{|l|}{ Posse de automóvel (\%) } \\
\hline Não possui & 59,3 & 69,6 & \\
\hline 1 & 31,5 & 26,1 & $>0,05^{2}$ \\
\hline $2 \mathrm{ou}+$ & 9,2 & 4,3 & \\
\hline
\end{tabular}

Nota: foi utilizado o teste Exato de Fischer ${ }^{\prime}$ e Qui quadrado ${ }^{2}$ para verificar a diferença entre os grupos para as respostas avaliadas, considerando-se o número de indivíduos, onde as respostas nenhuma e não possui foram isoladas das demais.

Tabela 4 - Respostas relativas às questões sobre acesso a serviços odontológicos em adultos e idosos. Piracicaba, 2002.

\begin{tabular}{llll}
\hline \multirow{2}{*}{ Variáveis } & \multicolumn{3}{c}{ Grupos } \\
\cline { 2 - 3 } & adultos & idosos \\
\hline
\end{tabular}

Já foi ao dentista $(\%)$

$\begin{array}{lccc}\text { Sim } & 96,3 & 100 & >0,05^{1} \\ \text { Não } & 3,7 & - & \end{array}$

Quanto tempo (\%)

$\begin{array}{cccc}\text { Nunca foi } & - & 4,3 & \\ \text { Menos de } 1 \text { ano } & 46,3 & 34,8 & >0,05^{2} \\ \text { De } 1 \text { a } 2 \text { anos } & 14,8 & 4,3 & \\ 3 \text { ou }+ \text { anos } & 38,9 & 56,5 & \end{array}$

Teve informações de como evitar problemas bucais $(\%)$

$\begin{array}{llll}\text { Sim } & 77,8 & 56,5 \\ \text { Não } & 22,2 & 43,5 & >0,05^{2}\end{array}$

Considera necessidade de tratamento $(\%)$

$\begin{array}{lll}\text { Sim } & 79,6 & 73,9 \\ \text { Não } & 20,4 & 26,1\end{array} \quad>0,05^{2}$

Nota: foi utilizado o teste Exato de Fischer ${ }^{1}$ e Qui quadrado ${ }^{2}$ para verificar a diferença entre os grupos para as respostas avaliadas, considerando-se o número de indivíduos, onde a respostas não e nunca foi, foram isoladas das demais. de 28,2 em 2002, sendo de 27,0 em Piracicaba, ou seja, em todos estes estudos, a saúde bucal dos idosos pode ser considerada como insatisfatória quando comparada com a dos adultos. No Brasil, os dados não foram diferentes, o CPOD para os adultos foi de 20,1 (65,7\% de dentes perdidos) e para os idosos, de 27,8 (92,9\% de dentes perdidos) (BRASIL, 2004).

Vale acrescentar que em Piracicaba, o componente que contribuiu com maior porcentagem para o índice CPOD, tanto em adultos como em idosos, foram os dentes perdidos. No entanto, quanto à presença de dentes, os adultos apresentaram média de dentes presentes três vezes maior $(20,5)$ comparada com os ido$\operatorname{sos}(6,9)$. Salienta-se ainda que apesar das condições clínicas terem sido bem diferentes, a autopercepção da saúde bucal foi semelhante para adultos e idosos.

Além da autopercepção, os dados sobre o nível socioeconômico não foram diferentes em ambos os grupos (apenas com relação à escolaridade), também não houve diferença com relação ao acesso a serviços odontológicos, apesar dos adultos terem ido ao dentista com uma freqüência maior que os idosos, o que pode ter a influência da melhor escolaridade e assim pode-se inferir que a saúde bucal seja melhor para este grupo devido a estes fatores.

Dados da literatura nacional apontam para índices CPOD em idosos que variam de 26,6 a 30,6 , sendo o maior componente referente a dentes perdidos, com uma porcentagem entre $77,2 \%$ e $96,3 \%$, onde a média de dentes presentes não ultrapassou 6,98 (SILVA, 1999; CARNEIRO, 2001; SILVA et al., 2002; SILVA et al., 2004), não apresentando diferenças notáveis quanto ao presente estudo.

A divulgação de dados sobre condições de saúde bucal em adultos é limitada, assim, dos trabalhos existentes, a situação não se difere do presente estudo, com CPOD variando de 20,4 a 22,9 , onde a perda dentária contribuiu substancialmente na experiência de cárie, com porcentagens entre 40,5\% a 50,0\% (CANGUSSU et al., 2001; FRAZÃO et al.; 2003, SILVA et al., 2004).

Uma medida de grande importância para a saúde bucal refere-se ao número de dentes presentes. Considerando-se que uma das metas da OMS para o ano 2000, era de que $50 \%$ dos idosos e $75 \%$ dos adultos apresentassem 20 dentes presentes ou mais, pôde-se observar que os percentuais apresentados neste estudo (13,0\% e 64,8\% de idosos e adultos, respectivamente) estão distantes de tais metas (PAIX $\tilde{A} O, 2002)$.

Os dados evidenciaram o problema de predominância de dentes perdidos, aumentando com isto o número de idosos edêntulos e mostrando a real necessidade da existência de programas específicos para estes grupos etários. Um fato importante a ser ressaltado neste estudo foi quanto à diferença apresentada entre as médias de dentes hígidos e presentes em adultos e idosos, onde os idosos apresentaram grande número de dentes perdidos com rela- 
Tabela 5 - Respostas relativas às questões sobre autopercepção em saúde bucal em adultos e idosos. Piracicaba, 2002.

\begin{tabular}{|c|c|c|c|}
\hline \multirow{2}{*}{ Variáveis } & \multicolumn{2}{|c|}{ Grupos } & \multirow{2}{*}{$\mathbf{P}$} \\
\hline & adultos & idosos & \\
\hline \multicolumn{4}{|l|}{ Saúde bucal (\%) } \\
\hline Péssima/ruim & 31,5 & 17,3 & \\
\hline Regular & 33,3 & 34,9 & $>0,05^{2}$ \\
\hline Boa/ótima & 35,2 & 47,8 & \\
\hline
\end{tabular}

\begin{tabular}{cccc}
\hline $\begin{array}{c}\text { Aparência dentes e } \\
\text { gengivas (\%) }\end{array}$ & & & \\
\hline Péssima/ruim & 31,5 & 21,7 & \\
Regular & 29,6 & 30,5 & $>0,05^{2}$ \\
Boa/ótima & 38,9 & 47,8 & \\
\hline
\end{tabular}

\begin{tabular}{cccc}
\hline Mastigação $(\%)$ & & & \\
\hline Péssima/ruim & 24,1 & 26,1 & $>0,05^{2}$ \\
Regular & 22,2 & 34,8 & 39,1 \\
Boa/ótima & 53,7 & & \\
\hline Fala (\%) & 11,2 & 13,0 & \\
\hline Péssima/ruim & 20,3 & 26,2 & \\
Regular & 68,5 & 60,8 & \\
Boa/ótima & & & \\
\hline
\end{tabular}

Saúde bucal afeta

relacionamento com

outras pessoas $(\%)$

\begin{tabular}{cccc}
\hline Não afeta & 57,4 & 56,5 & $>0,05^{2}$ \\
Afeta & 42,6 & 43,5 & \\
\hline
\end{tabular}

\section{Quanto de dor seus dentes \\ e gengivas causaram nos últios 6 meses $(\%)$}

$\begin{array}{lccc}\text { Nenhuma } & 53,7 & 60,9 & >0,05^{2} \\ \text { Pouca a muita } & 46,3 & 39,1 & \end{array}$

Nota: foi utilizado o teste Exato de Fischer ${ }^{1}$ e Qui quadrado ${ }^{2}$ para verificar a diferença entre os grupos para as respostas avaliadas, considerando-se o número de indivíduos, onde as respostas péssima/ruim/regular, não afeta e nenhuma, foram isoladas das demais. ção aos adultos. Este acontecimento pode estar relacionado à ida com maior freqüência ao dentista pelos adultos, o que implicaria em maiores cuidados. Por outro lado, ressalta-se o fato preocupante de a maioria dos idosos terem ido ao dentista há 3 anos ou mais, pois isto implica em não haver diagnóstico precoce principalmente de lesões em mucosa.

Com relação ao uso de prótese total, FRARE et al. (1997) em Pelotas -RS, relataram que $64,6 \%$ dos idosos eram edêntulos, onde a maioria usava prótese total superior por motivos estéticos e não usava inferior por apresentar desconforto, mostrando que há uma deficiência mastigatória, mesmo com o uso de próteses. Da amostra de idosos de Piracicaba, $52,2 \%$ eram edêntulos, sendo que também houve uma maior porcentagem dos que usavam próteses totais superiores.

Quanto ao uso e necessidade de próteses totais, os resultados deste estudo com relação aos idosos, não diferiram dos apresentados por CARNEIRO (2001), onde 48,12\% usavam próteses totais superiores e $22,53 \%$ inferiores e 36,18\% necessitavam de prótese total superior e $50,86 \%$ de inferior.

Os dados de 1998 sobre idosos e adultos moradores da região de Piracicaba (SÃO PAULO, 1998), apontaram que $91 \%$ dos idosos e $5 \%$ dos adultos necessitavam de prótese total superior e $75 \%$ e $3 \%$ de inferior, já os que usavam prótese total, a porcentagem foi de $98 \mathrm{e}$ 50 para a superior e 91 e 33 para a inferior, ou seja, a porcentagem de uso e necessidade de próteses totais foi maior que a relatada em $\mathrm{Pi}$ racicaba no ano de 2002 , ou seja, no presente estudo.

Diante destes resultados, torna-se importante mais uma vez enfatizar que ações de saúde bucal preventivas, curativas e reabilitadoras são necessárias, para que dessa forma possa haver adequadamente a promoção de saúde, com indicações específicas para cada grupo etário. Recomenda-se dar prioridade ao controle de doenças, bem como à complementação dos dados clínicos com dados sobre a percepção da saúde bucal, para que futuramente possa haver um maior número de idosos com seus dentes naturais e que os adultos possam permanecer com um número ainda maior de dentes presentes. Apesar dos dados clínicos apresentarem-se discrepantes entre os grupos, o mesmo não aconteceu com os dados sobre a percepção e acesso a serviços relativos a saúde bucal, que podem ser considerados positivos em ambos os grupos.

\section{CONCLUSÕES}

Houve uma alta prevalência de edentulismo nos idosos, com média de 6,9 dentes presentes, sendo 5,0 hígidos, já as condições de saúde bucal dos adultos diferiram, apresentando-se em melhores condições, porém as diferenças foram apenas referentes às condições clínicas e escolaridade. Torna-se necessário que o planejamento em saúde bucal para estes grupos tenham como base a realidade apresentada para que desta forma, sejam implementa- 
dos programas específicos que promovam saúde e consigam controlar doença, buscando efetivamente uma melhora na qualidade de vida.

\section{ABSTRACT}

This study investigated the oral health conditions, socioeconomic status, access to dental care and self-perception in oral health among elderly and adults from the city of Piracicaba (SP). The sample comprised 23 elderly individuals (65 to 74 years) and 54 adults (35 to 44 years). Examinations were performed at their homes, following the criteria of the World Health Organization. Edentulism was observed in $52.2 \%$ of the elderly and $2.0 \%$ of adults, and the mean number of present teeth was 6.9 and 20.7 , respectively. The elderly individuals presented DMFT of 27.0 and the adults 19.9. The most frequent component of the DMFT index was missing teeth for the elderly $(92.3 \%)$ and adults (52\%). Concerning the need of complete dentures, only $5.6 \%$ of the adults needed maxillary prostheses and there was no need of mandibular prostheses, whereas among the elderly $17.4 \%$ needed maxillary prostheses and $21.7 \%$ required mandibular prostheses. With regard to the other results (socioeconomic characteristics, access to dental care and self-perception), there was difference between groups only for the educational level, being that adults presented the best conditions. Self-perception was similar for both groups, with positive data, despite the different clinical conditions. Considering these results, there is the need of preventive and educational programs for both elderly and adults, so that the adults may be elderly individuals with better oral health status than the present.

\section{KEYWORDS:}

Adults, Elderly, Oral Health, Self-Perception, Oral health surveys.

\section{REFERÊNCIAS}

BRASIL. Ministério da Saúde. Secretaria de Atenção a Saúde. Departamento de Atenção Básica. Projeto SB 2003: Condições de Saúde Bucal da População Brasileira: 2002-2003. Brasília: Ministério da Saúde, 2004.

BRASIL. Ministério da Saúde. Pesquisa Nacional por Amostra de Domicílios (PNDA) 1998: Acesso e Utilização de Serviços de Saúde. Instituto Brasileiro de Geografia e Estatística. Ministério da Saúde, 1998.

BRASIL. Ministério da Saúde. Divisão Nacional de Saúde Bucal. Levantamento Epidemiológico em Saúde Bucal. Brasília: Ministério da Saúde, 1988.

CANGUSSU, M.C.T.; COELHO, E.O.; CASTELLANOS FERNANDEZ, R.A. Condições de Saúde Bucal em Adultos e Idosos em Itatiba/SP, Brasil -2000.
Rev. Odontol. Unesp, São Paulo, v. 30 , n. 2 , p. $245-256$, jul.-dez. 2001.

CARNEIRO, RMV. Saúde Bucal em Idosos Institucionalizados na Cidade de São Paulo: Estudo Epidemiológico e de Autopercepção. 2001. 111f. Dissertação (Mestrado em Saúde Pública) - Faculdade de Saúde Pública, Universidade de São Paulo, São Paulo.

FRARE, S.M. et al. Terceira Idade: Quais os Problemas Orais Existentes? Rev. Assoc. Paul. Cir. Dent., São Paulo, v. 51, n. 6, p. 573-576, nov./dez 1997.

FRAZÃO, P.; ANTUNES, J.L.F.; NARVAI, P.C. Perda Dentária Precoce em Adultos de 35 a 44 Anos de Idade. Estado de São Paulo, Brasil, 1998. Rev. Bras. Epidemiol., São Paulo, v. 6, n 1., p. 49-57, abr. 2003.

FUNDACÃO SISTEMA ESTADUAL DE ANÁLISE DE DADOS (Seade). Condições de Vida - Índice de Desenvolvimento Humano de Piracicaba e do Estado de São Paulo, 2005. Disponível em : <http://www.seade.gov.br > Acesso em: 16 de maio. 2005.

PAIXÃO, D. F. Tecnologia para Todos. Rev. Assoc. Paul. Cir. Dent., São Paulo, v. 56, n. 6 , p. 408-417, nov./dez. 2002.

PAPALlÉO NETTO, M.; PONTE, J. R. Envelhecimento: Desafio na Transição do Século. In: PAPALLÉO NETTO Matheus. Gerontologia. São Paulo: Atheneu. 2002. Cap. 1, p. 3-12.

PERES M.A. et al. The Association Between Socioeconomic Development at the Town Level and the Distribution of Dental Caries in Brazilian Children. Rev. Panam. Salud Publica, Kansas, v. 14, n. 3, p. 149-157, Sept. 2003.

PETERSEN, P. E. The World Oral Health Report 2003: Continuous Improvement of Oral Health in the 21st Century - The Approach of the WHO Global Oral Health Programme. Community Dent. Oral Epidemiol., Copenhagen, v. 31 Suppl.1, p. 3-23, Dec. 2003.

PINTO, V.G. Saúde Bucal para Adultos. In:__. Saúde Bucal Coletiva. 4 ed. São Paulo: Santos, 2000. Cap. 4 p. 114-116.

SÃO PAULO. Secretaria de Estado de Saúde de São Paulo (SES-SP). Levantamento Epidemiológico em Saúde Bucal: Estado de São Paulo, 1998. São Paulo: SES-SP, 1999.
SÃO PAULO. Secretaria de Estado de Saúde de São Paulo (SES-SP). Condições de Saúde Bucal: Estado de São Paulo, Direção Regional de Saúde de Piracicaba - DIR XV, 1998. São Paulo: SES-SP, 1998.

SÃO PAULO. Secretaria de Estado de Saúde de São Paulo (SES-SP). Condições de Saúde Bucal no Estado de São Paulo em 2002. São Paulo: SES-SP, 2002.

SHINKAI, R. S. A.; DEL BEL CURY, A. A. O Papel da Odontologia na Equipe Interdisciplinar: Contribuindo para a $\mathrm{Ob}$ tenção Integral ao Idoso. Cad. Saude Publica, Rio de Janeiro, v. 16, n. 4, p. 1099 1109, out./dez. 2000.

SILVA, D.D. et al. Condições de Saúde Bucal em Idosos na Cidade de Piracicaba. Rev. Assoc. Paul. Cir. Dent., São Paulo, v. 56, n.3, p. 183-187, maio/jun. 2002.

SILVA, D.D.; SOUSA, M.L.R.; WADA, R.S. Saúde Bucal em Adultos e Idosos na Cidade de Rio Claro, São Paulo, Brasil. Cad. Saude Publica, Rio de Janeiro, v. 20, n. 2, p. 626-631, mar./abr. 2004.

SILVA, SRC. Autopercepção das Condições Bucais em Pessoas com 60 Anos e mais de Idade. 1999. 116 f Tese (Doutorado em Saúde Pública). Faculdade de Saúde Pública, Universidade de São Paulo, São Paulo.

WORLD HEALTH ORGANIZATION (WHO). Oral Health Surveys: Basic Methods. Geneva: 1987.

WORLD HEALTH ORGANIZATION (WHO). Oral Health Surveys: Basic Methods. Geneva: 1997.

Endereços para correspondência: Silva, D. D.

Avenida Limeira, 901. Piracicaba - SP. Brasil. CEP 13414-018. Fax:(19) 21065218; Fone: 2106 5209. diasdeb@yahoo.com.br

Carvalho, O. L.

Avenida Limeira, 901. Piracicaba - SP. Brasil. CEP 13414-018. Fax: (19) 21065218; Fone: 2106 5209. shekina5@uol.com.br

Sousa, M. L. R.

Avenida Limeira, 901. Piracicaba - SP. Brasil. CEP 13414-018. Fax: (19)21065218; Fone:21065209. luzsousa@fop.unicamp.br

Hebling, E.

Avenida Limeira, 901. Piracicaba - SP. Brasil. CEP 13414-018. Fax: (19) 21065218; Fone:21065209. hebling@fop.unicamp.br 